\title{
SISTEMA DE GERENCIAMENTO DE IRRIGAÇÃO (SIGI): CÁLCULO DA EVAPOTRANSPIRAÇÃ̃O
}

\author{
Vanessa Robalo dos Santos, Marta Breunig Loose e Luís Henrique Loose \\ Instituto Federal Farroupilha \\ RS 218 - Km 5 - Bairro Indúbras - CEP 98806-700 - Santo Ângelo/RS - Brasil
}

\begin{abstract}
RESUMO
A irrigação tem grande importância na agricultura, pois auxilia a manter uma produção constante em diferentes cultivos. Em consequência disso o uso da água é um fator indispensável. Para amenizar o desperdício desse recurso, este artigo apresenta uma etapa do desenvolvimento de um sistema usado para controlar a irrigação de plantações em pequenas propriedades, denominado SiGi. Atualmente o sistema calcula e apresenta o resultado da evapotranspiração diária das plantas, cálculo esse que é essencial na definição da quantidade de água necessária para cada tipo de cultivo. No decorrer do artigo serão apresentados os parâmetros para realizar o cálculo, bem como as tecnologias utilizadas na codificação do sistema. O objetivo do SiGi é fornecer ao pequeno produtor uma tecnologia adequada para o efetivo desenvolvimento da plantação. O sistema apresenta como resultados os cadastros dos dados da propriedade, dos cultivos, das plantações, das precipitações diárias, bem como fornece diariamente o resultado da evapotranspiração de referência.
\end{abstract}

\section{PALAVRAS-CHAVE}

Irrigação, Sistema Web, Evapotranspiração, Programação

\section{INTRODUÇÃO}

A agricultura irrigada é uma ferramenta importante para suprir a grande demanda de produção de alimentos, devido ao aumento da população. Atualmente a agricultura irrigada produz mais de $40 \%$ dos alimentos do planeta (FAO, 2017). O processo de irrigar uma produção requer um estudo detalhado, pois existem muitas variáveis a serem consideradas antes de simplesmente fornecer água para a plantação. Encaixam-se nessas variáveis: as condições meteorológicas, a disponibilidade de água da região, o tamanho total da área a ser irrigada, o tipo de cultivo, o solo, dentre outros fatores que devem ser considerados. O manejo da irrigação, desde que bem planejado, pode trazer inúmeros benefícios para a plantação, sendo capaz de trazer aumento na produtividade, melhoria na qualidade dos produtos agrícolas, além de trazer maiores lucros ao produtor.

A disponibilidade de água é um dos principais fatores que torna possível o uso da irrigação. De acordo com a Agência Nacional das Águas (ANA, 2017), estima-se que o Brasil possua cerca de 12\% da disponibilidade de água doce do planeta. Analisando esses dados e comparando ao grande aumento populacional, cresce cada vez mais a necessidade do uso racional e sustentável de água em todos os setores da economia. Além disso, no Brasil a irrigação tornou-se um fator importante contra a escassez de água em determinados períodos do ano, em que normalmente não ocorrem chuvas frequentes. Um sistema de informação que auxilie no controle da irrigação dos cultivos agrícolas, permite ao produtor irrigar na quantidade exata que a planta necessita, garantindo a qualidade, uma boa produtividade e, ainda, evitando o desperdício de água.

Nesse sentido, este trabalho visa apresentar o SiGi Sistema de Gerenciamento de Irrigação em sua primeira etapa de implementação finalizada. O sistema já apresenta o resultado da evapotranspiração de referência diária, além de possibilitar o cadastro da propriedade, dos cultivos, das plantações e das precipitações diárias. 


\subsection{Evapotranspiração}

A evapotranspiração e a precipitação pluvial são os fatores determinantes para estimar a quantidade de irrigação necessária (Bernardo et al., 2006, p. 48). Para calcular a evapotranspiração, é necessária uma série de parâmetros relacionados à: condições meteorológicas, como a temperatura, a umidade do ar, a velocidade do vento, a radiação solar e a quantidade de chuva; ao solo, como a profundidade e a textura; e ao cultivo, como dossel vegetativo, a fase do ciclo e a época de cultivo. Segundo a FAO (1998) "a evapotranspiração (ET) é a combinação de dois processos separados pelos quais a água é perdida, por um lado a evaporação direta da superfície do solo e por outro lado, a transpiração das plantas”.

Existem vários métodos para determinar a evapotranspiração (Paiva \& De Souza, 2016), mas o método Penman-Monteith é definido como o método padrão para cálculo da ETo (FAO, 1998). Para determinar a ETo é necessário calcular algumas equações. Sendo o primeiro cálculo o da tangente à curva de saturação do ar (S), o segundo cálculo corresponde à Pressão de Saturação do ar, a terceira equação a ser calculada é a Pressão de vapor, posteriormente calcula-se o saldo de radiação solar ( $\mathrm{Q}^{*}$ ). Após a obtenção desses resultados calcula-se a evapotranspiração, através da equação de Penman-Monteith, mostrada na figura 1.

$$
E T_{0}=\frac{0.408\left(R_{n}-G\right)+\gamma \frac{900}{T+273} u_{2}\left(e_{s}-e_{a}\right)}{\Delta+\gamma\left(1+0.34 u_{2}\right)}
$$

Figura 1. Equação de Penman-Monteith para cálculo da evapotranspiração

Onde:

ETo: evapotranspiração de referência [mm dia -1 ];

Rn: radiação líquida na superfície da cultura [MJ m -2 dia -1 ];

$\mathrm{T}$ : temperatura do ar diariamente à 2 metros de altura $\left[{ }^{\circ} \mathrm{C}\right]$;

$\mathrm{U} 2$ : velocidade do vento à 2 metros de altura [ms -1 ];

es - ea: é a diferença de pressão de vapor de saturação [ $\mathrm{kPa}]$;

$\Delta$ : curva de pressão de vapor da inclinação $\left[\mathrm{kPa}{ }^{\circ} \mathrm{C}-1\right]$;

G: fluxo de calor no solo [5\% da Rn];

$\gamma$ : constante psicrométrica $\left[\mathrm{kPa}^{\circ} \mathrm{C}-1\right](\mathrm{FAO}, 1998)$

\subsection{Trabalhos Relacionados}

O Sistema Irriga ${ }^{\circledR}$ é um sistema de manejo e monitoramento de irrigação, criado e mantido por pesquisadores da Universidade Federal de Santa Maria (Viero, 2009, p. 51). Para fornecer a quantidade de água necessária na irrigação, esse sistema conta com estações meteorológicas automáticas, que fornecem dados diários das condições meteorológicas de cada local monitorado. São disponibilizados planos de contratação, com diferentes funcionalidades e serviços, sendo que a área irrigada mínima deve ser de 25 hectares.

Já o SisCI é um sistema usado para monitorar remotamente a irrigação através de dispositivos celulares (Almeida, Fernandes \& Costa, 2012). A plataforma é composta por um conjunto de sensores que captam os dados meteorológicos do campo irrigado e envia via wireless para uma aplicação web denominada SisCI Servidor. Já o SisCI Cliente permite ao usuário o acesso aos dados do seu campo irrigado.

Considerando os trabalhos relacionados, o $\mathrm{SiGi}$, sistema apresentado neste artigo possui como diferencial o atendimento a produtores rurais de pequenas áreas cultivadas. Além disso, o sistema auxiliará na irrigação de cultivos como hortaliças e frutíferas, que não são atendidos pelos demais sistemas disponíveis no mercado.

\section{IMPLEMENTAÇÃO DO SISTEMA}

O SiGi foi desenvolvido através do Framework Laravel. Usando da estrutura MVC (Model, View Controller), o Laravel utiliza o Composer para gerenciar suas dependências. Além disso, existe um 
gerenciamento padrão das rotas, onde através delas são chamadas as visões do sistema, sendo que a comunicação é direta com o respectivo controlador.

O layout da aplicação é definido através do Blade templates, que está acoplado ao framework, sendo que as visões usam a extensão do arquivo “.blade.php” (Laravel, 2018). O Blade usa uma sintaxe específica, deixando o código organizado e com uma estrutura padronizada. O Laravel conta também com o Artisan, que é uma interface de linhas de comandos que fornece várias instruções úteis e práticas na criação da aplicação. Já a interação com o banco de dados é realizada através do Eloquent ORM. A criação das tabelas passa pela utilização das migrations, que funcionam como controles de versões do banco de dados, sendo possível a realização de ajustes e compartilhamentos de maneira efetivamente fácil (Laravel, 2018).

\subsection{Upload de Dados}

Conforme explicado anteriormente, o cálculo da evapotranspiração é realizado através de uma série de variáveis relacionadas aos elementos meteorológicos. Em função disso torna-se necessária a coleta de dados de uma estação meteorológica mais próxima. O INMET (Instituto Nacional de Meteorologia) possui diversas estações automáticas distribuídas pelo país, as quais fornecem diariamente dados relativos aos elementos meteorológicos, disponibilizados através do site oficial (INMET 2018).

A estação automática da qual são coletados os dados necessários para o presente projeto está situada na cidade de Santa Rosa, estado do Rio Grande do Sul, Brasil. O armazenamento no banco de dados do SiGi é efetuado através do upload de arquivo gerado diariamente. Para isso, utiliza-se o Laravel Excel, que simplifica as importações e exportações de arquivo, além de um formulário simples. Na Figura 2 é mostrada a tela do SiGi com os dados que foram coletados do site da estação meteorológica e armazenados na base de dados. Será apresentada somente parte das telas nas figuras, em função do espaço limitado.

\begin{tabular}{|c|c|c|c|c|c|c|}
\hline Home $\mathrm{F}$ & Propriedades Glebas & Cultivos Plantaçðes & Chuvas Dado & eteorológicos Evapotra & de Referânc & \\
\hline \multicolumn{7}{|c|}{ Dados Meteorológicos } \\
\hline Código & Data & Temperatura & Umidade & Velocidade do Vento & Radiação & Volume de Chuva \\
\hline 1 & $01 / 06 / 2019$ & 17.33 & 83.50 & 1.44 & 12051.02 & 0 \\
\hline 2 & $02 / 06 / 2019$ & 15.69 & 85.83 & 1.60 & 10324.85 & 0.2 \\
\hline 3 & $03 / 06 / 2019$ & 15.01 & 88.33 & 1.44 & 10929.53 & 0.2 \\
\hline 4 & $04 / 06 / 2019$ & 14.55 & 78.92 & 1.75 & 13468.25 & 0.2 \\
\hline 5 & $05 / 06 / 2019$ & 13.88 & 70.00 & 1.68 & 13034.96 & 0.2 \\
\hline
\end{tabular}

Figura 2. Tela do sistema exibindo os dados meteorológicos armazenados

Os dados armazenados através do upload são referentes às 24 horas do dia, o que gera uma inserção de 24 linhas no banco de dados. Para o uso desses dados na equação citada na seção 1.1 é necessário um valor único para cada coluna em determinada data. Sendo assim, após o upload diário calcula-se, através de uma Função Armazenada, a média da temperatura, da umidade relativa do ar, da velocidade do vento, bem como a soma da radiação e da chuva.

\subsection{Cálculo da Evapotranspiração}

Após a execução do procedimento armazenado, o próximo passo é finalizar o cálculo da evapotranspiração. Para chegar a esse resultado é necessário calcular separadamente cada variável composta no método FAO Penman-Monteith, sendo elas: pressão de saturação de vapor de água no ar, pressão parcial de vapor de água no ar, tangente de curva de saturação no ar e saldo de radiação. Após a realização desses cálculos obtém-se o resultado da evapotranspiração de referência diária (Figura 3). 


\begin{tabular}{|c|c|c|c|c|c|}
\hline Home & Propriedades & s Glebas Cultivos & Plantaçøes & Chuvas Dados Meteorológicos & Evapotranspiração de Referência \\
\hline \multicolumn{6}{|c|}{ Evapotranspiraçäo de Referência (Eto) } \\
\hline Códig & & Data - Eto & Eto & Tipo de Cultivo & Data - Dados Meteorológicos \\
\hline 1 & & 01/06/2019 & 1.17 & Frutiferas & $01 / 06 / 2019$ \\
\hline 2 & & $02 / 06 / 2019$ & 1.01 & Frutiferas & $02 / 06 / 2019$ \\
\hline 3 & & $03 / 06 / 2019$ & 0.94 & Frutiferas & $03 / 06 / 2019$ \\
\hline 4 & & 04/06/2019 & 1.12 & Frutiferas & $04 / 06 / 2019$ \\
\hline 5 & & 05/06/2019 & 1.17 & Frutiferas & 05/06/2019 \\
\hline
\end{tabular}

Figura 3. Tela de exibição do resultado da evapotranspiração (ETo)

\subsection{Funcionalidades do SiGi}

O acesso ao sistema é realizado através de um formulário de login, em que o usuário informa seu e-mail e senha. Previamente, é necessário que o produtor rural realize seu cadastro, através da opção "Registre-se". Além de fornecer o resultado da ETo, o SiGi conta com outras funcionalidades disponíveis na tela inicial, como cadastros da propriedade, volume de chuva, cultivos, data da plantação e glebas, que de acordo com o dicionário Michaelis, "são terrenos apropriados para cultivo".

As telas de cadastros são compostas por formulários simples, já a tabela de exibição dos dados cadastrados permite a edição ou exclusão de registros. Como exemplo, a figura 4 mostra a tela de cadastro do volume de chuva para uma determinada data. O campo propriedade será preenchido automaticamente após a realização do login do produtor.

\begin{tabular}{l} 
Home Propriedades Glebas Cultivos Plantaçōes Chuvas Dados Meteorológicos Evapotranspiraçäo de Referência \\
Data \\
$\mathrm{dd} / \mathrm{mm} /$ aaaa \\
Volume de Chuva \\
Chuva \\
Propriedade \\
Selecione \\
Salvar Cancelar \\
\hline
\end{tabular}

Figura 4. Tela de cadastro do volume de chuva na propriedade

Além do cadastro do volume de chuva, também é possível visualizar um relatório dos dados armazenados. Conforme mostrado na figura 5, esse relatório apresenta a data, o volume de chuva e a propriedade, sendo possível editar ou apagar esses dados. No exemplo, é mostrado o relatório para o usuário administrador do sistema.

Os demais cadastros do SiGi, tais como propriedade, plantações e glebas, seguem o mesmo padrão de design nas suas telas. Além disso, as telas de exibição dos resultados também estão disponíveis para esses cadastros, sendo que é possível editar e apagar os mesmos. 


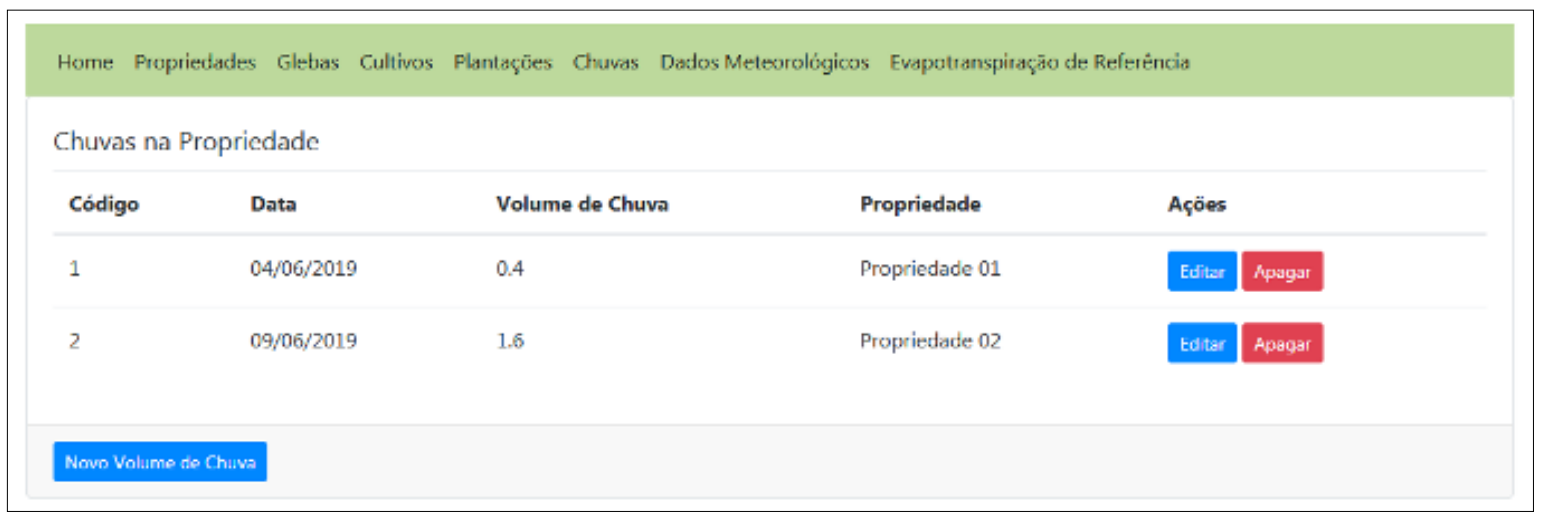

Figura 5. Tela de exibição dos volumes de chuva cadastrados na propriedade

\section{CONCLUSÃO}

O presente trabalho atingiu seus objetivos propostos, já que o sistema desenvolvido conta com as funcionalidades de cadastros da propriedade, dos cultivos, das plantações, das glebas e do volume de chuva, sendo uma importante ferramenta de gerenciamento ao produtor rural. A principal contribuição do $\mathrm{SiGi}$ até o momento é a realização do cálculo da evapotranspiração de referência, fornecendo um resultado preciso para auxiliar na definição da quantidade de água para a irrigação dos cultivos. Esta definição será parte da próxima etapa de desenvolvimento do sistema, através da implementação do cálculo do balanço hídrico. Dessa maneira, o presente trabalho continuará auxiliando o produtor rural, facilitando o gerenciamento da irrigação dos cultivos, visando a economia de água e o aumento na produtividade.

\section{REFERÊNCIAS}

Almeida, I. L.; Fernandes, C. F. I.; Costa, M. C. SISCI - Sistema para Controle de Irrigação através de Dispositivos Celulares. Holos, v. 1, p. 147-156. 2012. ISSN: 1807-1600. Disponível em: < http://www2.ifrn.edu.br/ojs/index.php/HOLOS/article/view/681>. Acesso em: 01 mai. 2018.

ANA - Agência Nacional das Águas. Atlas Irrigação: Uso da Água na Agricultura Irrigada. Brasília, 2017. Disponível em: < http://arquivos.ana.gov.br/imprensa/publicacoes/AtlasIrrigacao-UsodaAguanaAgriculturaIrrigada.pdf > . Acesso em: 23 abr. 2018.

Bernardo, S. et al, 2006. E. Manual da Irrigação. 8. ed. Viçosa: Ed. UFV.

Getcomposer.Com. Introdução. Disponível em:< https://getcomposer.org/doc/00-intro.md>. Acesso em: 30 set. 2018.

FAO - Organização das Nações Unidas para a Alimentação e Agricultura. Agricultura Irrigada Sustentável no Brasil: Identificação de áreas prioritárias. Brasília, 2017. Disponível em: < http://www.fao.org/3/a-i7251o.pdf>. Acesso em 29 abr. 2018.

FAO - Organização das Nações Unidas para a Alimentação e Agricultura. Evapotranspiração das culturas - Diretrizes para o cálculo das necessidades de água das culturas - Irrigação e drenagem da FAO 56. Roma, 1998. Disponível em < http://www.fao.org/docrep/X0490E/X0490E00.htm>. Acesso em: 30 abr. 2018.

INMET. Instituto Nacional de Meteorologia - Estações Automáticas. Brasilia, 2018. Disponível em: <http://www.inmet.gov.br/portal/index.php?r=estacoes/estacoesAutomaticas>. Acesso em: 24 jun. 2018.

Laravel.Com. Laravel: documentação. Disponível em: https://laravel.com/docs/5.6. Acesso em: 30 set. 2018.

Paiva, C. M., \& De Souza, A. D. S. P. (2016). Avaliação de métodos de estimativa da evapotranspiração de referência para fins de manejo da irrigação. Anuário do Instituto de Geociências, 39(1), 42-51.

Sistema Irriga. Sistema Irriga - Tecnologia UFSM. Santa Maria, 2018. Disponível em < https://www.sistemairriga.com.br/?servico=tecnologia>. Acesso em: 01 mai. 2018.

Viero, C. V. Tecnologias de Informação e Comunicação no contexto rural brasileiro: O modelo de Monitoramento agrícola do Sistema Irriga ${ }^{\circledR}$ Santa Maria, 2009. Disponível em: http://repositorio.ufsm.br/bitstream/handle/1/8836/VIERO\%2c\%20VERONICA\%20CRESTANI.pdf?sequence=1\&is Allowed=y>. Acesso em: 01 mai. 2018. 